\title{
Toward Autonomous Quantum Communication
}

\author{
A machine-learning algorithm previously used to solve navigation \\ problems can devise efficient ways to transmit quantum information.
}

\author{
By Marric Stephens
}

V achine-learning techniques are now routine in many fields of physics, where their uses include the prediction of novel materials (see Synopsis: Discovering New Magnetic Materials with Machine Learning) and the stabilization of synchrotrons (see Synopsis: Noisy synchrotron? Machine Learning Has the Answer). Now, Julius Wallnöfer, at the Free University of Berlin and colleagues use a machine-learning method to develop protocols for quantum communication [1]. They show that an autonomous agent equipped with a basic quantum toolkit can discover efficient combinations of operations for transmitting quantum states. The researchers hope that the approach could be used to optimize future quantum-communication networks.

Wallnöfer and colleagues use a learning agent that is known to perform well at solving navigation problems, which they say is analogous to devising quantum-communication protocols. The agent begins each task by randomly performing operations chosen from a "universal gate set"-a resource that allows any

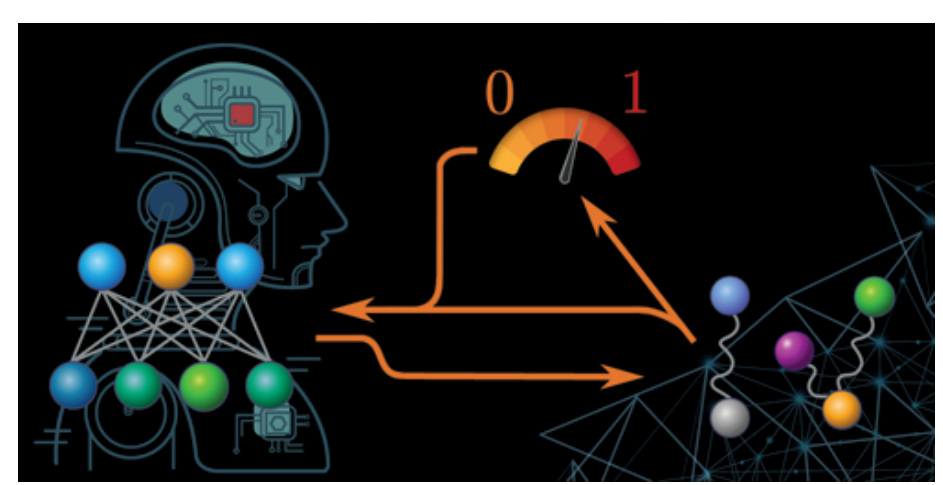

Credit: A. A. Melnikov/Free University of Berlin/University of Basel/Russian Academy of Sciences possible quantum operation given the right sequence of actions. The agent learns to achieve its goal with increasing efficiency by receiving rewards for actions that yield the desired outcome.

The agent can also combine previously learned operations to accomplish increasingly complex tasks. This ability lets the agent progress from devising quantum teleportation protocols, to developing entanglement-purification operations, to designing quantum repeaters for long-distance communication. The researchers find that the agent is especially effective at finding protocols when the individual sections of a communication channel have different levels of noise-a problem for which researchers still lack a robust solution.

How much autonomy such learning agents might one day wield is, the researchers say, an open question. In its current form, the agent is restricted to performing the role of an assistant-specifying the problems and defining the success criteria are still manual processes.

Marric Stephens is a Corresponding Editor for Physics based in Bristol, UK.

\section{REFERENCES}

1. J. Wallnöfer et al., "Machine learning for long-distance quantum communication," PRX Quantum 1, 010301 (2020). 